\title{
Tuberculosis en la edad avanzada
}

\section{Tuberculosis in advanced age}

LILIANA FERNÁNDEZ T., MD. ${ }^{(1)}$

El incremento de la expectativa de vida y la disminución de la tasa de nacimientos en el mundo, ha aumentado la población de edad avanzada, especialmente en los países desarrollados; este se convierte en un grupo vulnerable por las comorbilidades, la disfunción en la inmunidad celular, la malnutrición y los cambios relacionados con la edad, haciéndolo más susceptible a agentes infecciosos como el Mycobacterium tuberculosis. En 2015, 10,4 millones de personas fueron diagnosticadas con tuberculosis activa y de ellos 1,8 millones murieron.

La población de adultos mayores se convierte además en un gran reservorio de infección tuberculosa, usualmente con retardo en el diagnóstico pues en esta población la norma es la presentación atípica de la enfermedad que confunde y retarda el diagnóstico y aunque el manejo estándar tetraconjugado acortado supervisado anti-TBC es altamente efectivo, también se asocia en este grupo con una cantidad de no despreciable de efectos adversos relacionados con los medicamentos.
En este número se publica un artículo original donde se describe la experiencia con tuberculosis en pacientes mayores de 80 años atendidos es una institución de alta complejidad. Entre los años 2011 y 12016 , se diagnosticaron 27 pacientes mayores de 80 años con factores de riesgo relacionados como enfermedad pulmonar obstructiva crónica (EPOC), tabaquismo, diabetes, cáncer, entre otros. En el $74 \%$ de los casos se presentó retardo en el diagnóstico mayor de treinta días, 52\% tuvieron TBC pulmonar en los cuales el $41 \%$ tuvo baciloscopias positivas. Se describe un preocupante $37 \%$ de pérdidas en el seguimiento que pueden estar relacionadas con el fraccionamiento de la atención en nuestro sistema de salud. La forma extrapulmonar fue más frecuente en los más ancianos.

La incidencia de tuberculosis entonces aumenta exponencialmente en los individuos con edad avanzada y está ligada a un desenlace desfavorable con tratamientos más dispendiosos, más largos y con más efectos adversos.
(1)Medicina Interna, Neumología, Neumología Intervencionista. Línea de Investigación Biomédica en Tórax, Fundación Valle del Lili. Profesora Clínica Asociada, Facultad de Ciencias de la Salud, Universidad Icesi. Cali, Colombia. Correspondencia: Liliana Fernández T., correos electrónicos: 1fernandez@fcvl.org lilianafernandeztrujllo@gmail.com Recibido: 15/05/17. Aceptado: 20/05/17. 